\title{
Intravitreal Ranibizumab in the Treatment of Butterfly-Shaped Pattern Dystrophy Associated with Choroidal Neovascularization: A Case Report
}

\author{
Theodoros Empeslidis Athanasios Vardarinos James Deane \\ Somnath Banerjee \\ Leicester Royal Infirmary, University Hospitals of Leicester, Leicester, UK
}

\section{Key Words}

Intravitreal ranibizumab - Pattern dystrophy of the macula $\cdot$ Choroidal neovascular membrane $\cdot$ Choroidal neovascularization

\begin{abstract}
Purpose: To present and document the effectiveness of intravitreal ranibizumab in the treatment of patients with choroidal neovascularization due to butterfly-shaped pattern dystrophy (PD) of the macula.

Methods: Three intravitreal ranibizumab injections of $0.5 \mathrm{mg} / 0.05 \mathrm{ml}$ in monthly intervals were given to a patient with a previously diagnosed butterfly-shaped PD who subsequently developed subfoveal choroidal neovascularization on the right eye. The patient had previously received a combination of verteporfin/photodynamic therapy for a juxtafoveal choroidal neovascular membrane on the left eye.
\end{abstract}

Results: At the end of the treatment course, there was significant improvement of the patient's vision and the appearance of the macula on optic coherence tomography and fluorescein angiography. Best-corrected visual acuity improved from 6/12 to $6 / 6$ and retinal thickness at the macula decreased from 323 to $247 \mu \mathrm{m}$. No subretinal fluid remained. The patient is clinically stable over a 12-month follow-up period.

Conclusions: Intravitreal ranibizumab seems to be an effective and safe option for the treatment of subfoveal choroidal neovascularization in patients with butterfly-shaped PD. 


\section{Introduction}

Pattern dystrophies (PD) are a heterogeneous group of inherited disorders of the retinal pigment epithelium which usually appear in middle life, affecting both eyes, predominantly at the macula and are considered to have a chronic progressive course, although in most patients functional vision is preserved in later life. Butterfly-shaped PD was initially described by Zhang et al. [1] as a relatively benign pigmentary dystrophy of the fovea affecting the retinal pigment epithelium. It has been linked to mutations on the RDS/peripherin gene on chromosome $6 \mathrm{p} 21$. Although uncommon, the disease may progress significantly over time and some patients might develop visual impairment due to atrophic changes or the development of a choroidal neovascular membrane (CNVM) at the macula $[1,2]$.

\section{Case Presentation}

In 2004, a 58-year-old female patient presented at the retina clinic with symptoms of mild bilateral distortion, especially of the left eye, and difficulty in reading. Clinical examination, fundus fluorescein angiogram (FFA), and electrophysiology testing were performed. The electroretinogram was normal, but the electrooculogram was subnormal. The diagnosis of butterfly-shaped PD was established due to clinical phenotype and investigation results (fig. 1). On the left eye, the FFA revealed a juxtafoveal CNVM and was treated with a combination of verteporfin and photodynamic therapy (PDT) which, at the time, was considered to be the best treatment option. After the treatment, the CNVM was obliterated, and the patient was stabilized and put on a regular follow-up until his discharge in 2007.

In 2010, the patient returned to the retina clinic with decreased vision and distortion in the right eye this time. On examination, best-corrected visual acuity (BCVA) on a Snellen chart was 6/12 in the right eye and 6/6 in the left. The FFA and the optical coherence tomography (OCT) revealed the presence of an active subfoveal CNVM in the right eye with accumulation of subretinal fluid and increased retinal thickness (323 $\mu \mathrm{m})$ (fig. 2). A decision was made to treat the patient with a course of three-monthly intravitreal ranibizumab injections $(0.5 \mathrm{mg} / 0.05 \mathrm{ml})$.

The patient gave her informed consent for all material and medical records presented in this case report.

\section{Results}

Following the course of three-monthly intravitreal ranibizumab injections of 0.5 $\mathrm{mg} / 0.05 \mathrm{ml}$, the retinal thickness decreased from 323 to $247 \mu \mathrm{m}$ on OCT scan images, and no evidence of subretinal fluid or intraretinal fluid could be revealed clinically or on OCT scan (fig. 3 ). The repeated FFA did not reveal any late-phase hyperfluoroscence due to leakage from the CNVM. The patient's BCVA improved from 6/12 to 6/9 (Snellen chart) immediately after the 3-monthly intravitreal ranibizumab injections of 0.05 $\mathrm{mg} / 0.05 \mathrm{ml}$.

The patient was monitored in 4-6 weeks intervals with an OCT scan and clinical assessment was performed. Four months after the last intravitreal injection, the patient's BCVA had improved to 6/6. He remained asymptomatic with no reactivation of the CNVM for over 12 months following the last ranibizumab intravitreal injection. The fellow eye which had previously been treated with PDT developed a juxtafoveal 
atrophic scar, and the BCVA remained stable at 6/6 (Snellen chart). Interestingly, the patient's BCVA seemed to improve the weeks following the last injection. We have observed similar outcomes in several patients treated with anti-vascular endothelial growth factor (anti-VEGF) injections for age-related CNMV in our department, and a possible explanation is that anatomical or OCT improvement could precede functional visual improvement in these patients.

\section{Discussion}

PDs are a group of retinal disorders which predominantly affect the retinal pigment epithelium and unless complicated with the development of atrophy or CNMV do not cause serious visual impairment [2,3]. Previously, verteporfin/PDT was utilized for the treatment of CNMV in patients with PD. Results from these attempts showed that although PDT had a temporary advantageous effect on visual function, the thermal insult to the retinal pigment epithelium cells would inevitably lead to the development of a significant atrophic scar which eventually would cause long-term deterioration of vision $[3,4]$.

Promising results were recently published regarding the use of intravitreal bevacizumab for the treatment of subfoveal CNVM associated with PD. Parodi et al. [5] studied a series of patients who were treated with a course of intravitreal bevacizumab for a period of more than 2 years and managed to document significant and sustainable improvement in terms of both BCVA and retinal thickness. That study added further data to earlier reports of an advantageous effect of bevacizumab in PD-associated CNVM [6]. Furthermore, Gallego-Pinazo et al. [7] very recently published a series of female patients diagnosed with adult-onset foveomacullar vitelliform dystrophyrelated CNVM in whom short-term promising results regarding BCVA were documented.

In our case, the use of a standard loading dose of three intravitreal ranibizumab injections in a female patient with subfoveal CNVM due to butterfly-shaped PD led to a remarkable improvement in vision from $6 / 12$ to $6 / 6$ (Snellen chart), significant reduction of retinal thickness, and a normalization of the macular appearance on OCT. The fellow eye which was previously treated with PDT for a juxtafoveal CNVM developed an atrophic scar; however, vision remained stable and satisfactory $(6 / 6$ on Snellen chart).

It is well documented in the literature that CNVM due to non-age-related macular degeneration conditions, such as in high myopia, respond better when treated with anti-VEGF intravitreal injections $[8,9]$. Our patient remains stable with no evidence of reactivation of the condition 12 months following the last intravitreal ranibizumab 0.5 $\mathrm{mg} / 0.05 \mathrm{ml}$ ) injection and there is also a number of small series of non-age-related CNVM where even one intravitreal anti-VEGF injection was satisfactory to control the CNVM and stabilize the patient's vision for a substantial amount of time [10,11]. The patient is undergoing frequent monitoring (every 2 months) and although clinically stable up to now, recurrence of the condition cannot be excluded.

This report adds some more valuable data to the recently published promising good results regarding the use of bevacizumab and ranibizumab in other PD-related CNVM. 
To the best of our knowledge, this is the first report on the use of ranibizumab in butterfly-shaped PD-related CNVM. With the current evidence, it seems that both bevacizumab and ranibizumab are reliable and effective choices for the treatment of subfoveal PD-related CNVM as an alternative to the previously used combination of verteporfin/PDT. However, longer follow-up time, higher number of patients, as well as safety profile assessments are needed in future studies.

\section{Disclosure Statement}

None of the authors has conflict of interest to disclose. No financial support was received for this submission.

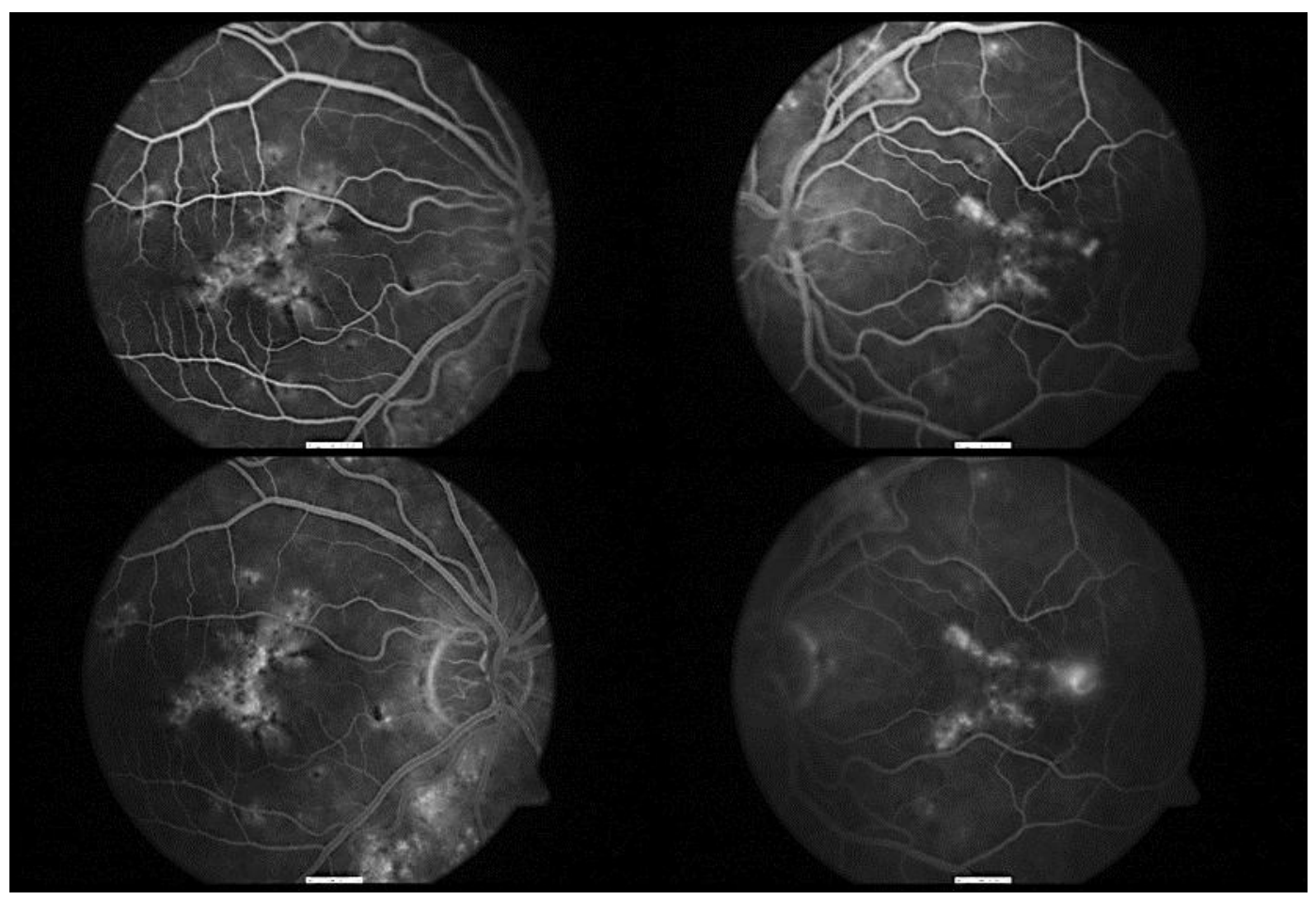

Fig. 1. FFA showing the typical butterfly pattern dystrophy. 


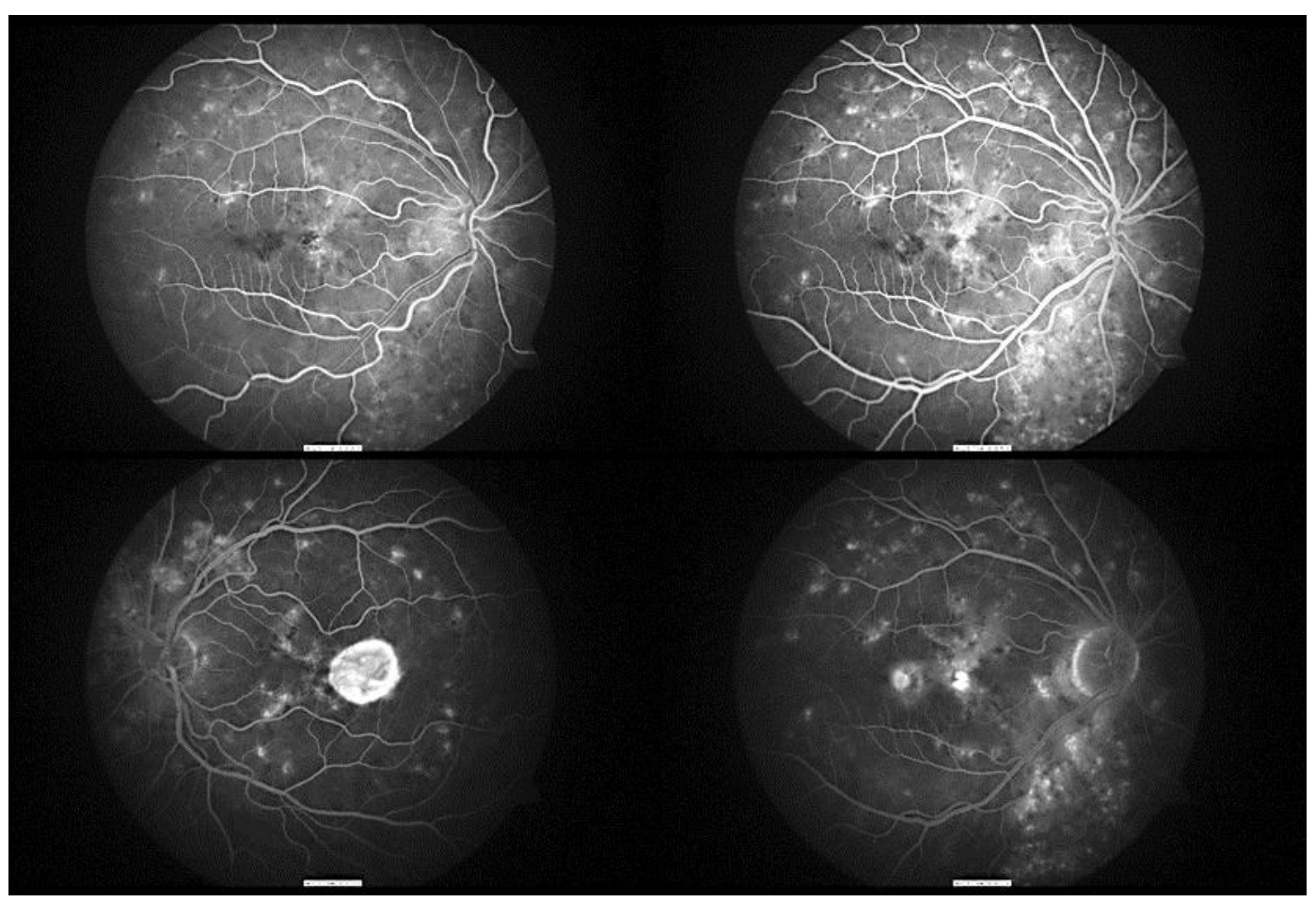

Fig. 2. Right eye showing late leakage on FFA due to CNVM and left eye showing a well demarcated area of atrophy temporarily to the left macula as a result of PDT.

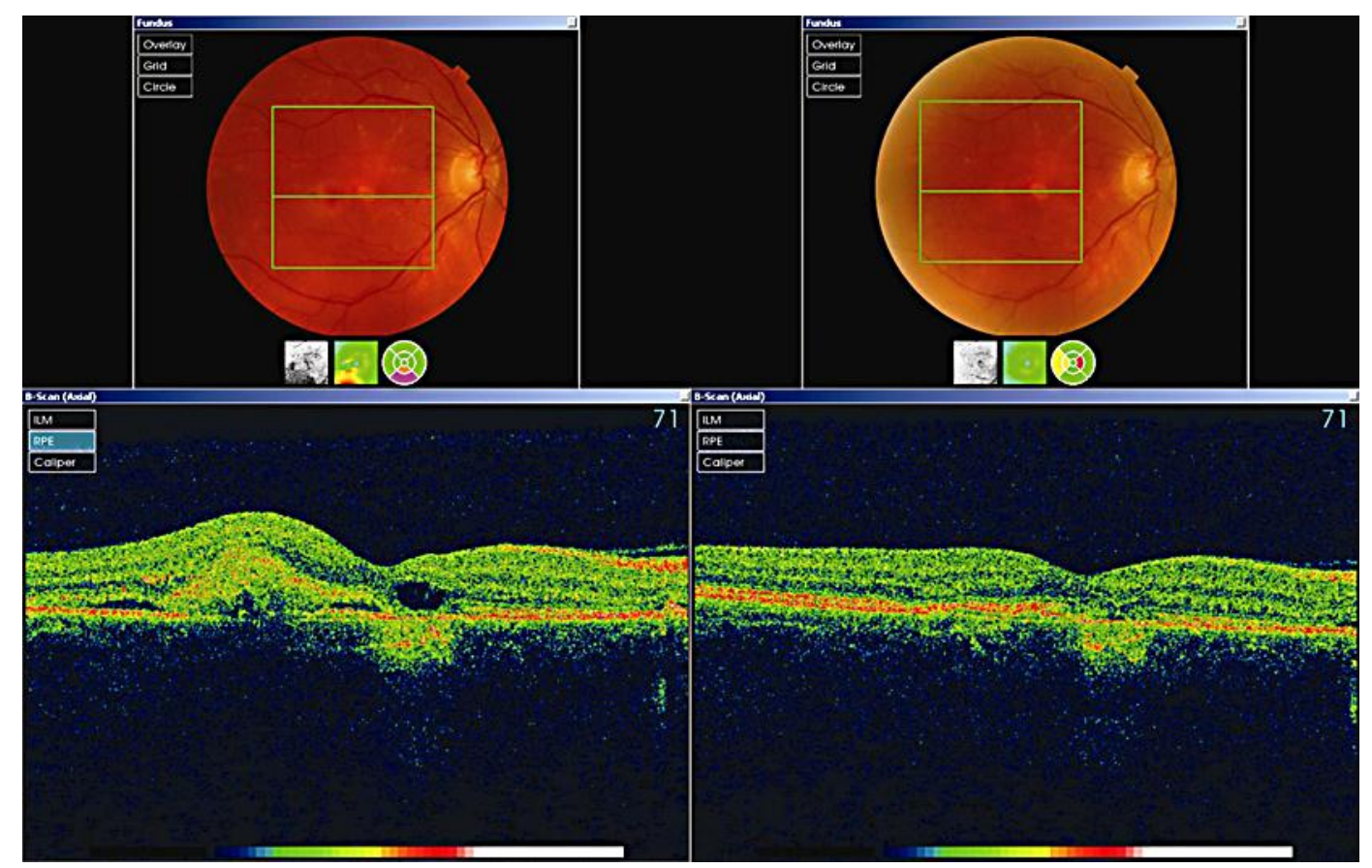

Fig. 3. OCT on the right before the course of anti-VEGF treatment and OCT on the left following a course of three anti-VEGF intravitreal injections. 


\section{References}

1 Zhang K, Garibaldi DC, Yang L, Grenn WR: Butterfly-shaped pattern dystrophy. A genetic, clinical and histopathological report. Arch Ophthalmolol 2002;120:485-490.

-2 Marano F, Deutman AF, Leys A, Aandekerk AL: Hereditary retinal dystrophies and choroidal neovascularization. Graefes Arch Clin Exp Ophthalmol 2000;238:760-764.

-3 Parodi MB, Da Pozzo S, Ravalico G: Photodynamic therapy for choroidal neovascularization associated with pattern dystrophy. Retina 2003;23:171-176.

4 Parodi MB, Liberally T, Pedio M, et al: Photodynamic therapy for subfoveal choroidal neovascularization secondary to reticular pattern dystrophy: three-year results of an uncontrolled, prospectivecase series. Am J Ophthalmol 2006;141:1152-1154.

-5 Parodi MB, Iacono P, Cascavilla M, Zucchiatti I, Kontadakis DS, Bandello F: Intravitreal bevacizumab for subfoveal choroidal neovascularization associated with pattern dystrophy. Invest Ophthalmol Vis Sci 2010;51:4358-4361.

-6 Montero JA, Ruiz-Moreno JM, De La Vega C: Intravitreal bevacizumab for adult-onset vitelliform dystrophy: a case report. Eur J Ophthalmol 2007;17:983-986.

-7 Gallego-Pinazo R, Dolz-Marco R, Pardo-Lopez D, Arevalo JF, Diaz-Llopis M: Primary intravitreal ranibizumab for adult-onset foveomacular vitelliform dystrophy. Graefes Arch Clin Exp Ophthalmol 2011;249:455-458.

8 Arias L, Planas N, Prades S: Intravitreal bevacizumab (Avastin) for myopic choroidal neovascularization. Br J Ophthalmol 2008;92:1035-1039.

9 Chan WM, Lai TY, Liu DT, Lam DS: Intravitreal bevacizumab (Avastin) for myopic choroidal neovascularisation: 1-year results of prospective pilot study. BR J Ophthalmol 2009;93:150-154.

10 Neri P, Salvolini S, Mariotti C, Mercanti L, Celani S, Giovannini A: Long-term control of choroidal neovascularisation secondary to angioid streaks treated with intravitreal bevacizumab (Avastin). $\mathrm{Br}$ J Ophthalmol 2009;93:155-158.

11 Wiegand TW, Rogers AH, McCabe F, Reichel E, Duker JS: Intravitreal bevacizumab (Avastin) treatment of choroidal neovascularisation in patients with angioid streaks. Br J Ophthalmol 2009;93:47-51. 